\title{
Quasi-invariant subspaces generated by polynomials with nonzero leading terms
}

\author{
by \\ Kunyu Guo (Shanghai) and Shengzhao Hou (Linfen and Jiaxing)
}

\begin{abstract}
We introduce a partial order relation in the Fock space. Applying it we show that for the quasi-invariant subspace $[p]$ generated by a polynomial $p$ with nonzero leading term, a quasi-invariant subspace $M$ is similar to $[p]$ if and only if there exists a polynomial $q$ with the same leading term as $p$ such that $M=[q]$.
\end{abstract}

1. Introduction. The Fock space, also called the Segal-Bargmann space, is the analog of the Bergman space in the context of the complex $n$-space $\mathbb{C}^{n}$. It is a Hilbert space consisting of entire functions in $\mathbb{C}^{n}$. Let

$$
d \mu(z)=e^{-|z|^{2} / 2} d v(z)(2 \pi)^{-n}
$$

be the Gaussian measure on $\mathbb{C}^{n}$ (here $d v$ is the ordinary Lebesgue measure). The Fock space $L_{a}^{2}\left(\mathbb{C}^{n}, d \mu\right.$ ) (for short, $L_{a}^{2}\left(\mathbb{C}^{n}\right)$ ) is, by definition, the space of all $\mu$-square-integrable entire functions on $\mathbb{C}^{n}$. It is easy to check that $L_{a}^{2}\left(\mathbb{C}^{n}\right)$ is a closed subspace of $L^{2}\left(\mathbb{C}^{n}\right)$ with the reproducing kernel function $K_{\lambda}(z)=e^{\bar{\lambda} z / 2}$ (here $\left.\bar{\lambda} z=\sum_{i=1}^{n} \bar{\lambda}_{i} z_{i}\right)$.

For the theory of the Fock space we mention the work of [Jan1, Jan2, JS, CS], where Toeplitz operators on the Fock space were investigated, which is related to our work in this note. The Fock space is important because of the relationship between the operator theory on it and the Weyl quantization $[B e]$. For the Fock space, unlike the Hardy space $H^{2}(\mathbb{D})$ and the Bergman space $L_{a}^{2}(\mathbb{D})$ over the open unit disk $\mathbb{D}$, Guo and Zheng [GZh] showed that there exists no nontrivial invariant subspace for polynomials. That is, if $p$ is a polynomial and $M$ is a closed subspace of $L_{a}^{2}\left(\mathbb{C}^{n}\right)$, then the relation $p M$ $\subset M$ implies that either $p$ is a constant or $M=\{0\}$. Thus, an appropriate substitute for invariant subspace, the so-called quasi-invariant subspace is

2000 Mathematics Subject Classification: 46J15, 46H25, 47A15.

The first author is partially supported by NNSFC (10171019), Shuguang project in Shanghai, Young Teacher Fund for Higher Education of the National Educational Ministry of China and the grant of STCSM (03JC14013). The second author is partially supported by NNSFC (10301019) and the Natural Science Foundation of Shanxi province (20021005). 
needed. Namely, a (closed) subspace $M$ of $L_{a}^{2}\left(\mathbb{C}^{n}\right)$ is called quasi-invariant if $p M \cap L_{a}^{2}\left(\mathbb{C}^{n}\right) \subset M$ for each polynomial $p$. In $[\mathrm{GZh}]$, it is proved that for each finite-codimensional ideal $I$ of the polynomial ring $\mathbb{C}\left[z_{1}, \ldots, z_{n}\right]$, the closure of $I$, denoted by $[I]$, is quasi-invariant. Furthermore, Guo [Guo3] proved that $[I]$ is quasi-invariant if $I$ is a homogeneous ideal. However it is not known whether $[I]$ is quasi-invariant for any ideal $I$. Moreover, following Douglas and Paulsen [DP] and Guo [Guo1, Guo2, Guo3] we are naturally concerned with classifying all quasi-invariant subspaces in a reasonable sense.

Let $M_{1}$ and $M_{2}$ be two quasi-invariant subspaces of $L_{a}^{2}\left(\mathbb{C}^{n}\right)$. A bounded linear operator $A: M_{1} \rightarrow M_{2}$ will be called a quasi-module map if $A(p f)=$ $p A(f)$ whenever $p f \in M_{1}$ (here $p$ is any polynomial, and $f \in M_{1}$ ). Furthermore, we say that

(1) $M_{1}$ and $M_{2}$ are unitarily equivalent if there exists a unitary quasimodule map $A: M_{1} \rightarrow M_{2}$ such that $A^{-1}: M_{2} \rightarrow M_{1}$ is also a quasi-module map;

(2) $M_{1}$ and $M_{2}$ are similar if there exists an invertible quasi-module map $A: M_{1} \rightarrow M_{2}$ such that $A^{-1}: M_{2} \rightarrow M_{1}$ is also a quasi-module map;

(3) $M_{1}$ and $M_{2}$ are quasi-similar if there exist quasi-module maps $A$ : $M_{1} \rightarrow M_{2}$ and $B: M_{2} \rightarrow M_{1}$ with dense ranges.

It is easy to check that unitary equivalence, similarity and quasi-similarity are equivalence relations.

In Section 2, we explore a partial order relation on the entire function ring $\operatorname{Hol}\left(\mathbb{C}^{n}\right)$ of the complex $n$-space $\mathbb{C}^{n}$. Let $f, g$ be entire functions on $\mathbb{C}^{n}$. We say $f \preceq g$ if there exist positive constants $r, M$ such that

$$
\left|f\left(z_{1}, \ldots, z_{n}\right)\right| \leq M\left|g\left(z_{1}, \ldots, z_{n}\right)\right|
$$

whenever $\left|z_{i}\right|>r$ for $i=1, \ldots, n$. Then we prove that $f \preceq g$ if and only if there exist a polynomial $p$ and another polynomial $q$ with nonzero leading term satisfying $\operatorname{deg}_{z_{i}} p \leq \operatorname{deg}_{z_{i}} q$ for $i=1, \ldots, n$ such that

$$
f / g=p / q \text {. }
$$

This result will be used in Section 3 to study the order structure of the Fock space. In Section 3, we prove that if $f \preceq g$ and $g \in L_{a}^{2}\left(\mathbb{C}^{n}\right)$, then $f \in L_{a}^{2}\left(\mathbb{C}^{n}\right)$. Applying this result in Section 4 it is shown that for the quasiinvariant subspace $[p]$ generated by a polynomial $p$ with nonzero leading term, a quasi-invariant subspace $M$ is similar to $[p]$ if and only if there exists a polynomial $q$ with the same leading term as $p$ such that $M=[q]$.

2. A partial order on the entire function ring $\operatorname{Hol}\left(\mathbb{C}^{n}\right)$. In $[\mathrm{CGH}]$, we proved the following proposition. 
Proposition 2.1. Let $f$ and $g$ be entire functions on $\mathbb{C}$. Then $f \preceq g$ if and only if there exist polynomials $p$ and $q$ with $\operatorname{deg} p \leq \operatorname{deg} q$ such that

$$
f / g=p / q \text {. }
$$

The purpose of this section is to generalize this result to the case of several variables, and it will be applied to the study of similarity of quasiinvariant subspaces of the Fock space.

For an entire function $f$ on $\mathbb{C}^{n}$, we write $Z(f)$ for the set of all $z \in \mathbb{C}^{n}$ at which $f(z)=0$.

To give the characterization of the relation " $\preceq$ " on the entire function ring $\operatorname{Hol}\left(\mathbb{C}^{n}\right)$, we need two lemmas from [Guo2].

LEMMA 2.2 ([Guo2]). Let $f=p / q$ be a rational function, where $p$ and $q$ have no common factor. If $f$ is analytic on $\Omega\left(\subset \mathbb{C}^{n}\right)$, then $Z(q) \cap \Omega=\emptyset$.

LEMma 2.3 ([Guo2]). Let $f$ be in the Nevanlinna class on the polydisk $\mathbb{D}^{n}$, and let the slice functions $f_{w}$ be rational (of one variable) for almost all $w \in \mathbb{T}^{n}$. Then $f$ is a rational function (of $n$ variables).

Let $p\left(z_{1}, \ldots, z_{n}\right)=\sum_{i_{j} \leq m_{j}} a_{i_{1} \ldots i_{n}} z_{1}^{i_{1}} z_{2}^{i_{2}} \ldots z_{n}^{i_{n}}$ be a polynomial. If $a_{m_{1} \ldots m_{n}}$ $\neq 0$, we say that the leading term of $p$ is nonzero. For a polynomial $p$, we use $\operatorname{deg}_{z_{i}} p$ to denote the degree of $p$ in the variable $z_{i}$ for $i=1, \ldots, n$. For $0<r<\infty$, set

$$
\Omega_{r}=\left\{\left(z_{1}, \ldots, z_{n}\right) \in \mathbb{C}^{n}:\left|z_{i}\right|>r \text { for } i=1, \ldots, n\right\} .
$$

The next proposition gives a geometric characterization of polynomials with nonzero leading terms.

Proposition 2.4. Let $p$ be a polynomial in $n$ variables. Then the leading term of $p$ is nonzero if and only if there exists a positive constant $r$ such that $p$ has no zero in $\Omega_{r}$, that is,

$$
Z(p) \cap \Omega_{r}=\emptyset .
$$

Proof. For simplicity, we only give the proof for $n=2$. If $p(z, w)=$ $\sum_{i \leq m, j \leq n} a_{i j} z^{i} w^{j}$ has nonzero leading term $a_{m n} z^{m} w^{n}$, then

$$
p(z, w)=z^{m} w^{n}\left(a_{m n}+\sum_{(i, j) \neq(m, n)} a_{i j} z^{i-m} w^{j-n}\right),
$$

and hence there exists a positive constant $r$ such that $p$ has no zero in $\Omega_{r}$. For the opposite direction, $\operatorname{let}_{\operatorname{deg}_{z}} p=m$ and $\operatorname{deg}_{w} p=n$. Write

$$
p(z, w)=\sum_{k=0}^{m} p_{k}(w) z^{m-k}=\sum_{l=0}^{n} p_{l}^{\prime}(z) w^{n-l} .
$$

Choose a large $R_{0}(>r)$ such that both $p_{0}(w) \neq 0$ and $p_{0}^{\prime}(z) \neq 0$ if both $|z| \geq R_{0}$ and $|w| \geq R_{0}$. Note that on the circle $\left\{w:|w|=R_{0}\right\}$, if $R\left(>R_{0}\right)$ 
is sufficiently large, then we have

$$
\left|p_{0}(w) R^{m}+p_{1}(w) R^{m-1}+\cdots+p_{m}(w)-p_{0}(w) R^{m}\right|<\left|p_{0}(w)\right| R^{m} .
$$

Note that the polynomial $p_{0}(w) R^{m}+p_{1}(w) R^{m-1}+\cdots+p_{m}(w)=p_{0}^{\prime}(R) w^{n}+$ $p_{1}^{\prime}(R) w^{n-1}+\cdots+p_{n}(R)$ has exactly $n$ zeros in the disk $\left\{w:|w|<R_{0}\right\}$ (counting multiplicity). Now Rouché's theorem [Con] shows that $p_{0}(w)$ has $n$ zeros (counting multiplicity), and hence $\operatorname{deg} p_{0}=n$. This completes the proof.

TheOREM 2.5. Let $f$ and $g$ be entire functions on $\mathbb{C}^{n}$. Then $f \preceq g$ if and only if there exist a polynomial $p$ and another polynomial $q$ with nonzero leading term satisfying $\operatorname{deg}_{z_{i}} p \leq \operatorname{deg}_{z_{i}}$ q for $i=1, \ldots, n$ such that

$$
f / g=p / q \text {. }
$$

Proof. " $\Rightarrow "$. For convenience, we give the proof in the case of two variables. For an entire function $f$ on $\mathbb{C}^{2}$ and $w \in \mathbb{T}^{2}$ (2-torus), the slice function $f_{w}$ on the complex plane $\mathbb{C}$ is defined by $f_{w}(\lambda)=f(\lambda w), \lambda \in \mathbb{C}$. Note that $f \preceq g$ means that there exist positive constants $r, M$ such that

$$
|f(z, w)| /|g(z, w)| \leq M
$$

for $|z|>r$ and $|w|>r$. Let $\left(z_{0}, w_{0}\right) \in \mathbb{C}^{2}$ be such that $g\left(z_{0}, w_{0}\right) \neq 0$. Then there is a constant $r_{0}>0$ such that $g(z, w)$ has no zero on

$$
\Delta_{r_{0}}=\left\{(z, w):\left|z-z_{0}\right| \leq 2 r_{0},\left|w-w_{0}\right| \leq 2 r_{0}\right\} .
$$

Set $f_{0}(z, w)=f\left(r_{0} z+z_{0}, r_{0} w+w_{0}\right)$ and $g_{0}(z, w)=g\left(r_{0} z+z_{0}, r_{0} w+w_{0}\right)$. Then $f_{0} \preceq g_{0}$. Set $F=f_{0} / g_{0}$. Then $F$ is analytic on the closed bidisk $\overline{\mathbb{D}^{2}}$ and hence it is in the Nevanlinna class on $\mathbb{D}^{2}$. Now for each fixed $(z, w) \in \mathbb{T}^{2}$,

$$
\left|F_{(z, w)}(\lambda)\right|=\left|f_{0}(\lambda z, \lambda w)\right| /\left|g_{0}(\lambda z, \lambda w)\right|<M
$$

if $|\lambda|>\left(r+\left|z_{0}\right|+\left|w_{0}\right|\right) / r_{0}$. Hence by Proposition 2.1 , there exist polynomials $h_{1}, h_{2}$ in one variable such that

$$
F_{(z, w)}=h_{1} / h_{2} \text {. }
$$

Now by Lemma 2.3 , there are polynomials $p_{0}$ and $q_{0}$ without common factor such that

$$
F_{(z, w)}=f_{0}(z, w) / g_{0}(z, w)=p_{0}(z, w) / q_{0}(z, w),
$$

and hence there are polynomials $p$ and $q$ without common factor such that

$$
f / g=p / q \text {. }
$$

From the relation $p \preceq q$ and Proposition 2.1, one easily obtains

$$
\operatorname{deg}_{z} p \leq \operatorname{deg}_{z} q, \quad \operatorname{deg}_{w} p \leq \operatorname{deg}_{w} q .
$$

To obtain the desired conclusion, it is enough to show $q(z, w)$ has nonzero leading term. We claim

$$
Z(q) \cap \Omega_{r}=\emptyset .
$$


In fact, note that

$$
|p(z, w)| /|q(z, w)|<M \quad \text { if }(z, w) \in \Omega_{r} .
$$

Then by $[\mathrm{KK}]$, the function $p(z, w) / q(z, w)$ is analytic on $\Omega_{r}$. Now Lemma 2.2 implies that the claim is true, and Proposition 2.4 shows that $q$ has nonzero leading term.

"६". Let $q=\sum_{i \leq m, j \leq n} a_{i j} z^{i} w^{j}$ with nonzero leading term $a_{m n} z^{m} w^{n}$ and $p=\sum_{i \leq m, j \leq n} b_{i j} z^{i} w^{j}$. Note that

$$
p / q=\frac{\sum_{i \leq m, j \leq n} b_{i j} z^{i-m} w^{j-n}}{a_{m n}+\sum_{(i, j) \neq(m, n)} a_{i j} z^{i-m} w^{j-n}} .
$$

From the above equality, it is easy to see that there exist positive constants $r, M$ such that $|f(z, w)| /|g(z, w)|<M$ whenever $(z, w) \in \Omega_{r}$, i.e. $f \preceq g$. This completes the proof.

For entire functions $f, g$ on $\mathbb{C}^{n}$, if $f \preceq g$, then by Theorem 2.5 , there exist polynomials $p$ and $q$ without common factor such that $f / p=g / q$. Set

$$
h=f / p=g / q .
$$

Then $h$ is analytic on $\mathbb{C}^{n}-Z(p) \cap Z(q)$. Because $p$ and $q$ have no common factor, the set $Z(p) \cap Z(q)$ is a removable singularity for analytic functions $[\mathrm{KK}]$. This means that $h$ is analytic on all $\mathbb{C}^{n}$. So, we have the following conclusion.

Corollary 2.6. Let $f, g$ be entire functions on $\mathbb{C}^{n}$. Then $f \preceq g$ if and only if there exist a polynomial $p$, another polynomial $q$ with nonzero leading term satisfying $\operatorname{deg}_{z_{i}} p \leq \operatorname{deg}_{z_{i}} q$ for $i=1, \ldots, n$, and an entire function $h$ such that

$$
f=p h, \quad g=q h .
$$

We say that $f \sim g$ if $f \preceq g$ and $g \preceq f$. Note that " $\sim$ " is an equivalence relation on $\operatorname{Hol}\left(\mathbb{C}^{n}\right)$. From Theorem 2.5, we get the following corollary.

Corollary 2.7. Let $f, g \in \operatorname{Hol}\left(\mathbb{C}^{n}\right)$. Then $f \sim g$ if and only if there exist polynomials $p$ and $q$ with the same nonzero leading term such that $f / g=p / q$.

3. The order structure of the Fock space. Let $f, g$ be entire functions on $\mathbb{C}$. If $g \in L_{a}^{2}(\mathbb{C})$ and $f \preceq g$, then it is easy to verify that $f \in L_{a}^{2}(\mathbb{C})$. However, in the case of several variables, this fact is not obvious.

THEOREM 3.1. Let $f, g$ be entire functions on $\mathbb{C}^{n}$. If $f \preceq g$ and $g \in$ $L_{a}^{2}\left(\mathbb{C}^{n}\right)$, then $f \in L_{a}^{2}\left(\mathbb{C}^{n}\right)$.

Proof. For simplicity, we prove the theorem in the case of two variables. Since $f \preceq g$, there exist positive constants $r, M$ such that $|f| \leq M|g|$ on 
$\Omega_{r}=\{(z, w):|z|>r,|w|>r\}$. This implies that

$$
\int_{\Omega_{r}}|f(z, w)|^{2} d \mu<\infty .
$$

Set

$$
\begin{aligned}
& \Omega_{r}^{1}=\{(z, w):|z| \leq r,|w| \leq r\}, \quad \Omega_{r}^{2}=\{(z, w):|z| \leq r,|w|>r\} \\
& \Omega_{r}^{3}=\{(z, w):|z|>r,|w| \leq r\} .
\end{aligned}
$$

Then obviously,

$$
\int_{\Omega_{r}^{1}}|f(z, w)|^{2} d \mu<\infty
$$

Below we verify

$$
\int_{\Omega_{r}^{2}}|f(z, w)|^{2} d \mu<\infty .
$$

Let $f(z, w)=\sum_{m, n} a_{m n} z^{m} w^{n}$ be the power series expansion of $f$. Then

$$
\begin{aligned}
& \int_{\Omega_{r}}|f(z, w)|^{2} d \mu=\sum_{m, n}\left|a_{m n}\right|^{2} \int_{r}^{\infty} r_{1}^{2 m+1} e^{-r_{1}^{2} / 2} d r_{1} \int_{r}^{\infty} r_{2}^{2 n+1} e^{-r_{2}^{2} / 2} d r_{2} \\
& =\sum_{m, n} 2^{m+n}\left|a_{m n}\right|^{2} \int_{r^{2} / 2}^{\infty} x^{m} e^{-x} d x \int_{r^{2} / 2}^{\infty} x^{n} e^{-x} d x \\
& =\frac{1}{4} \sum_{m, n} r^{2(m+n+2)}\left|a_{m n}\right|^{2} \int_{1}^{\infty} t^{m} e^{-r^{2} t / 2} d t \int_{1}^{\infty} t^{n} e^{-r^{2} t / 2} d t \quad\left(x=r^{2} t / 2\right),
\end{aligned}
$$

and

$$
\begin{aligned}
& \int_{\Omega_{r}^{2}}|f(z, w)|^{2} d \mu=\sum_{m, n}\left|a_{m n}\right|^{2} \int_{0}^{r} r_{1}^{2 m+1} e^{-r_{1}^{2} / 2} d r_{1} \int_{r}^{\infty} r_{2}^{2 n+1} e^{-r_{2}^{2} / 2} d r_{2} \\
& =\sum_{m, n} 2^{m+n}\left|a_{m n}\right|^{2} \int_{0}^{r^{2} / 2} x^{m} e^{-x} d x \int_{r^{2} / 2}^{\infty} x^{n} e^{-x} d x \\
& =\frac{1}{4} \sum_{m, n} r^{2(m+n+2)}\left|a_{m n}\right|^{2} \int_{0}^{1} t^{m} e^{-r^{2} t / 2} d t \int_{1}^{\infty} t^{n} e^{-r^{2} t / 2} d t \quad\left(x=r^{2} t / 2\right) .
\end{aligned}
$$

Since

$$
\frac{\int_{0}^{1} t^{m} e^{-r^{2} t / 2} d t}{\int_{1}^{\infty} t^{m} e^{-r^{2} t / 2} d t} \leq \frac{\int_{0}^{1} e^{-r^{2} t / 2} d t}{\int_{1}^{\infty} e^{-r^{2} t / 2} d t}=e^{r^{2} / 2}-1,
$$

this gives

$$
\int_{\Omega_{r}^{2}}|f(z, w)|^{2} d \mu \leq\left(e^{r^{2} / 2}-1\right) \int_{\Omega_{r}}|f(z, w)|^{2} d \mu<\infty .
$$


The same reasoning yields

$$
\int_{\Omega_{r}^{3}}|f(z, w)|^{2} d \mu<\infty .
$$

We thus conclude

$$
\int_{\mathbb{C}^{2}}|f(z, w)|^{2} d \mu<\infty .
$$

This completes the proof of the theorem.

From Corollary 2.6 and Theorem 3.1, the next corollary is immediate.

Corollary 3.2. For entire functions $f, g$ on $\mathbb{C}^{n}$, if $f \preceq g$ and $g \in$ $L_{a}^{2}\left(\mathbb{C}^{n}\right)$, then there exist a polynomial $p$, another polynomial $q$ with nonzero leading term satisfying $\operatorname{deg}_{z_{i}} p \leq \operatorname{deg}_{z_{i}} q$ for $i=1, \ldots, n$, and a function $h \in L_{a}^{2}\left(\mathbb{C}^{n}\right)$ such that

$$
f=p h, \quad g=q h .
$$

4. Quasi-invariant subspaces generated by polynomials with nonzero leading terms. Let $p$ be a polynomial, and let $[p]$ be the closure of the principal ideal $p \mathbb{C}\left[z_{1}, \ldots, z_{n}\right]$. Then by [NS] or [Jan1, Jan2, JS], it is easy to check that $[p]$ is quasi-invariant. In this section we will prove that for the quasi-invariant subspace $[p]$ generated by a polynomial $p$ with nonzero leading term, a quasi-invariant subspace $M$ is similar to $[p]$ if and only if there exists a polynomial $q$ with the same leading term as $p$ such that $M=[q]$.

For convenience, in what follows we only consider the case of two variables, while all conclusions hold for $n$ variables.

Because the polynomial ring forms a core for Toeplitz operators with polynomial symbols (cf. [JS]) the next lemma is obvious.

LEMMA 4.1. For nonnegative integers $m, n$, we have

$$
\left[z^{m} w^{n}\right]=\left\{z^{m} w^{n} f \in L_{a}^{2}\left(\mathbb{C}^{2}\right): f \in \operatorname{Hol}\left(\mathbb{C}^{2}\right)\right\} .
$$

REMARK 4.2. By Theorem 3.1, the relation $f \preceq z^{m} w^{n} f \in L_{a}^{2}\left(\mathbb{C}^{2}\right)$ implies $f \in L_{a}^{2}\left(\mathbb{C}^{2}\right)$. It follows that

$$
\left[z^{m} w^{n}\right]=\left\{z^{m} w^{n} f \in L_{a}^{2}\left(\mathbb{C}^{2}\right): f \in L_{a}^{2}\left(\mathbb{C}^{2}\right)\right\} .
$$

Let $p(z, w)$ be a polynomial with leading term $z^{m} w^{n}$. Set

$$
L_{p}=\left\{p f \in L_{a}^{2}\left(\mathbb{C}^{2}\right): f \in \operatorname{Hol}\left(\mathbb{C}^{2}\right)\right\} .
$$

Then clearly, $L_{p}$ is quasi-invariant. Noting the relation $p f \sim z^{m} w^{n} f$ for any entire function $f$ we have

$$
L_{p}=\left\{p f \in L_{a}^{2}\left(\mathbb{C}^{2}\right): f \in L_{a}^{2}\left(\mathbb{C}^{2}\right)\right\} .
$$

Now by Corollary 2.7, Theorem 3.1 and the closed graph theorem, the following proposition is immediate. 
Proposition 4.3. There exists a similarity $A:\left[z^{m} w^{n}\right] \rightarrow L_{p}$, where $A z^{m} w^{n} f=p f$.

TheOREM 4.4. Let $p(z, w)$ be a polynomial with leading term $z^{m} w^{n}$. Then $[p]=L_{p}$.

Proof. Obviously, $[p] \subset L_{p}$. Now let $p f \in L_{a}^{2}\left(\mathbb{C}^{2}\right)$, and let $f=\sum_{s, t} a_{s t} z^{s} w^{t}$ be the power series expansion of $f$. Set

$$
f_{k l}=\sum_{s \leq k, t \leq l} a_{s t} z^{s} w^{t}
$$

for nonnegative integers $k, l$. By Proposition 4.3, there exist positive constants $C_{1}, C_{2}$ such that

$$
C_{1}\left\|z^{m} w^{n} h\right\| \leq\|p h\| \leq C_{2}\left\|z^{m} w^{n} h\right\|
$$

whenever $z^{m} w^{n} h \in L_{a}^{2}\left(\mathbb{C}^{2}\right)$. So, we have

$$
C_{1}\left\|z^{m} w^{n}\left(f-f_{k l}\right)\right\| \leq\left\|p f-p f_{k l}\right\| \leq C_{2}\left\|z^{m} w^{n}\left(f-f_{k l}\right)\right\| .
$$

Since

$$
\lim _{\substack{k \rightarrow \infty \\ l \rightarrow \infty}}\left\|z^{m} w^{n}\left(f-f_{k l}\right)\right\|=0
$$

we obtain

$$
\lim _{\substack{k \rightarrow \infty \\ l \rightarrow \infty}}\left\|p f-p f_{k l}\right\|=0
$$

and hence $p f \in[p]$. This gives the desired conclusion.

Before going on we need the following lemma. For a quasi-invariant subspace $M$, it is easy to see that $M \cap \mathbb{C}[z, w]$ is an ideal. The following lemma is essentially from $[\mathrm{CG}]$.

Lemma 4.5. Let $A: M_{1} \rightarrow M_{2}$ be a quasi-module map. Then $A$ maps $M_{1} \cap \mathbb{C}[z, w]$ to $M_{2} \cap \mathbb{C}[z, w]$. Moreover, for each nonzero $p \in M_{1} \cap \mathbb{C}[z, w]$, we have

$$
\operatorname{deg}_{z} q \leq \operatorname{deg}_{z} p, \quad \operatorname{deg}_{w} q \leq \operatorname{deg}_{w} p,
$$

where $q=A p$.

Proof. We may assume that $M_{1}$ contains a nonzero polynomial $p$. Set $q=A(p)$. We first claim that $\operatorname{deg}_{z} q \leq \operatorname{deg}_{z} p$. Suppose $\operatorname{deg}_{z} q>\operatorname{deg}_{z} p$. Then we expand $p$ and $q$ in the variable $z$ by

$$
p=p_{0}+p_{1} z+\cdots+p_{l} z^{l}, \quad q=q_{0}+q_{1} z+\cdots .
$$

Since $\operatorname{deg}_{z} q>\operatorname{deg}_{z} p$, there exists a positive integer $s(>l)$ such that $q_{s} \neq 0$. From the equality

$$
\left\|A\left(z^{k} p\right)\right\|^{2}=\left\|z^{k} q\right\|^{2}=\sum_{i=0}^{\infty}\left\|z^{k+i} q_{i}\right\|^{2},
$$


we have

$$
\left\|z^{k+s} q_{s}\right\|^{2} \leq\|A\|^{2} \sum_{i=0}^{l}\left\|z^{k+i} p_{i}\right\|^{2} .
$$

Since

$$
\left\|z^{k+s} q_{s}\right\|^{2}=\left\|z^{k+s}\right\|^{2}\left\|q_{s}\right\|^{2}=2^{k+s}(k+s) !\left\|q_{s}\right\|^{2}
$$

and

$$
\left\|z^{k+i} p_{i}\right\|^{2}=2^{k+i}(k+i) !\left\|p_{i}\right\|^{2}, \quad i=0,1, \ldots, l,
$$

for any natural number $k$, we infer that $q_{s}=0$. This yields the desired contradiction. The same reasoning implies $\operatorname{deg}_{w} q \leq \operatorname{deg}_{w} p$, and hence the conclusion follows.

From Lemma 4.5, the next corollary is immediate.

Corollary 4.6. Let $M$ be quasi-invariant. If there exists a quasi-module map $A:\left[z^{m} w^{n}\right] \rightarrow M$ with dense range, then $q=A z^{m} w^{n}$ is a polynomial in two variables with $\operatorname{deg}_{z} q \leq m$ and $\operatorname{deg}_{w} q \leq n$, and $M=[q]$.

Now we can prove our main result in this section.

TheOREM 4.7. Let $M$ be quasi-invariant. Then $\left[z^{m} w^{n}\right]$ and $M$ are quasisimilar if and only if there is a polynomial $q$ with leading term $z^{m} w^{n}$ such that $M=[q]$.

Proof. By Proposition 4.3 and Theorem 4.4, the sufficiency is obvious. Now if $\left[z^{m} w^{n}\right]$ and $M$ are quasi-similar, then there exist quasi-module maps $A:\left[z^{m} w^{n}\right] \rightarrow M$ and $B: M \rightarrow\left[z^{m} w^{n}\right]$ with dense ranges, and hence Corollary 4.6 gives $M=[q]$ for some polynomial $q$ with $\operatorname{deg}_{z} q \leq m$ and $\operatorname{deg}_{w} q \leq n$. From [GZh, Lemma 5.2], $[I] \cap \mathbb{C}[z, w]=I$ for each ideal $I$. Combining this fact with Lemma 4.5 and Corollary 4.6, we see that there is a nonzero constant $\gamma$ such that $B q=\gamma z^{m} w^{n}$ and $\operatorname{deg}_{z} q=m, \operatorname{deg}_{w} q=n$. Let $q(z, w)=q_{0}(w) z^{m}+q_{1}(w) z^{m-1}+\cdots+q_{m}(w)$ be the expansion of $q$ in the variable $z$. Note that

$$
\begin{aligned}
\left\|B z^{k} w^{l} q\right\|^{2}= & |\gamma|^{2}\left\|z^{m+k}\right\|^{2}\left\|w^{n+l}\right\|^{2} \\
\leq & \|B\|^{2}\left\|z^{k} w^{l} q\right\|^{2} \\
= & \|B\|^{2}\left(\left\|w^{l} q_{0}(w)\right\|^{2}\left\|z^{m+k}\right\|^{2}+\left\|w^{l} q_{1}(w)\right\|^{2}\left\|z^{m+k-1}\right\|^{2}\right. \\
& \left.+\cdots+\left\|w^{l} q_{m}(w)\right\|^{2}\left\|z^{k}\right\|^{2}\right) .
\end{aligned}
$$

Dividing the above inequality by $\left\|z^{m+k}\right\|^{2}$ and letting $k \rightarrow \infty$ gives

$$
|\gamma|^{2}\left\|w^{n+l}\right\|^{2} \leq\|B\|^{2}\left\|w^{l} q_{0}(w)\right\|^{2}
$$

for any positive integer $l$. This implies $\operatorname{deg} q_{0} \geq n$ and hence $\operatorname{deg} q_{0}=n$. So, $q(z, w)$ is a polynomial with leading term $z^{m} w^{n}$, completing the proof. 
Corollary 4.8. Let $M$ be a quasi-invariant subspace, and let $p(z, w)$ be a polynomial with leading term $z^{m} w^{n}$. Then the following statements are equivalent.

(1) $M$ is similar to $[p]$;

(2) $M$ is quasi-similar to $[p]$;

(3) there exists a polynomial $q(z, w)$ with leading term $z^{m} w^{n}$ such that $M=[q]$.

Combining Corollary 4.8 with the proof of Theorem 5.12 in [GZh], one can obtain

Corollary 4.9. Let $M$ be a quasi-invariant subspace, and let $p(z, w)$ be a polynomial with leading term $z^{m} w^{n}$. Then $M$ is unitarily equivalent to $[p]$ only if $M=[p]$.

Acknowledgements. The authors would like to thank Professor Xiaoman Chen for his encouragement and discussions. The authors also wish to express their gratitude to the referee for suggestions which helped make this paper more readable.

\section{References}

[Be] F. Berezin, Quantization, Math. USSR Izv. 8 (1974), 1109-1163.

[CG] X. M. Chen and K. Y. Guo, Analytic Hilbert Modules, Res. Notes in Math. 433, Chapman\&Hall/CRC, 2003.

[CGH] X. M. Chen, K. Y. Guo and S. Z. Hou, Analytic Hilbert spaces over the complex plane, J. Math. Anal. Appl. 268 (2002), 684-700.

[CS] D. Cichoń and J. Stochel, On Toeplitz operators in Segal-Bargmann spaces, Univ. Iagel. Acta Math. 34 (1997), 35-43.

[Con] J. Conway, Functions of One Complex Variable, Springer, 1973.

[DP] R. Douglas and V. Paulsen, Hilbert modules over Function Algebras, Longman, Harlow, 1989.

[Guo1] K. Y. Guo, Characteristic spaces and rigidity for analytic Hilbert modules, J. Funct. Anal. 163 (1999), 133-151.

[Guo2] - Equivalence of Hardy submodules generated by polynomials, ibid. 178 (2000), $343-371$.

[Guo3] - Homogeneous quasi-invariant subspaces of the Fock space, J. Austral. Math. Soc. 75 (2003), 399-407.

[GZh] K. Y. Guo and D. C. Zheng, Invariant subspaces, quasi-invariant subspaces, and Hankel operators, J. Funct. Anal. 187 (2001), 308-342.

[Jan1] J. Janas, Unbounded Toeplitz operators in the Bargmann-Segal space, Studia Math. 99 (1991), 87-99.

[Jan2] - Unbounded Toeplitz operators in the Segal-Bargmann space, III, Math. Scand. 88 (2001), 126-136.

[JS] J. Janas and J. Stochel, Unbounded Toeplitz operators in the Segal-Bargmann space, II, J. Funct. Anal. 126 (1994), 418-447.

[KK] L. Kaup and B. Kaup, Holomorphic Functions, de Gruyter, Berlin, 1983. 
[NS] D. Newman and H. Shapiro, Fischer spaces of entire functions, in: Entire Functions and Related Parts of Analysis, J. Koorevaar (ed.), Proc. Sympos. Pure Math. 11, Amer. Math. Soc., Providence, 1968, 360-369.

Department of Mathematics

Fudan University

Shanghai 200433

People's Republic of China

E-mail: kyguo@fudan.edu.cn
Department of Mathematics Shanxi Teachers University Linfen 041004

People's Republic of China

Current address of S. Z. Hou: Institute of Mathematics Jiaxing College Jiaxing 314001

People's Republic of China

E-mail: szhou@etang.com

Received March 19, 2002

Revised version January 13, 2003 